\title{
Poroid hidradenoma of the scalp
}

\author{
Byung Duk Min ${ }^{1}$, \\ Chong Kun Lee', \\ Chang Eun Chung ${ }^{1}$, \\ Dong Chul Kim ${ }^{1}$, \\ So Ya Paik ${ }^{2}$ \\ Departments of ${ }^{1}$ Plastic and \\ Reconstructive Surgery and ${ }^{2}$ Pathology, \\ Bundang Jesaeng Hospital, Seongnam, \\ Korea
}

\begin{abstract}
Poroid hidradenoma has both features of hidradenoma and poroma. The histological hidradenoma framework consisting of solid and cystic components, and the presence of poroid and cuticular cells resembling a poroid neoplasm. Despite transforming into malignant neoplasm only in $<1 \%$ of cases, its histological characteristics may resemble those of malignant neoplasms. Although the risk of malignant transformation is very low, surgical excision is recommended to prevent growth and/or recurrence. To date, very few cases of poroid hidradenoma have been reported in the literature. Herein, we present a case of poroid hidradenoma on the scalp of a 74-year-old woman.
\end{abstract}

Keywords: Eccrine glands / Poroma / Skin neoplasms

\section{INTRODUCTION}

Poroid hidradenoma is a benign neoplasm with eccrine differentiation, first described by Abenoza and Ackerman in 1990 [1]. These usually benign and asymptomatic neoplasms rarely become malignant. The age at onset ranges from 20 to 70 years old with a peak of incidence in the 7 th decade, but no observed sex differences. The neoplasm is typically well-circumscribed to the intradermal layer, with a diameter between 1 and $2 \mathrm{~cm}$, and a slightly reddish and round shape [2]. Although it is most commonly found in the trunk, it can also occur in the extremities, scalp, and face. There are few reported cases of poroid hidradenoma in the scalp. This study reports one such case in a woman in her seventies.

\section{CASE REPORT}

A 74-year-old woman presented to our hospital with a mass on her scalp, which she had for 40 years. When she first noticed the mass and she applied caustic soda at a beauty shop, about

Correspondence: Chong Kun Lee

Department of Plastic and Reconstructive Surgery, Bundang Jesaeng Hospital,

20 Seohyeon-ro 180beon-gil, Bundang-gu, Seongnam 13590, Korea

E-mail: chong5184@hanmail.net

Received March 25, 2021 / Revised April 20, 2021 / Accepted May 31, 2021
40 years prior and in the last 3 years the mass had rapidly grown showing ulceration. The patient had a complex medical history of hypertension, arrhythmia, and thyroid cancer after total thyroidectomy. Further, she was maintained on oral nifedipine for hypertension. Clinical assessments revealed a firm, fixed, protruding mass measuring about $2.0 \times 3.0 \times 0.5 \mathrm{~cm}$ in size with ulceration and pigmented nodular lesions (Fig. 1). The clinical diagnosis was nevus with pyogenic granuloma, and the lesion surgically excised. Gross examination revealed the tumor to be a fibrogranuloma. However, since the patient had previously applied caustic soda on the lesion and ulcer was observed overall, the frozen biopsy was decided to perform during surgery.

We performed duplicate tissue excision and frozen biopsy to confirm cell malignancy and local invasion (Fig. 2). The first simple excision and frozen biopsy during surgery revealed a poroid hidradenoma with atypia and abundant tumor cells at the anterior and posterior resection margins but not at the right and left sides (Fig. 3A). Since this biopsy could not rule out basal cell carcinoma, we performed wide excision with a safety margin of $5 \mathrm{~mm}$ (Fig. 3B). Then, the surgical wound was directly closed without complications. The pathology report confirmed poroid hidradenoma with cell atypia and free of all resection margins. Histopathologically, there was a well-circum- 


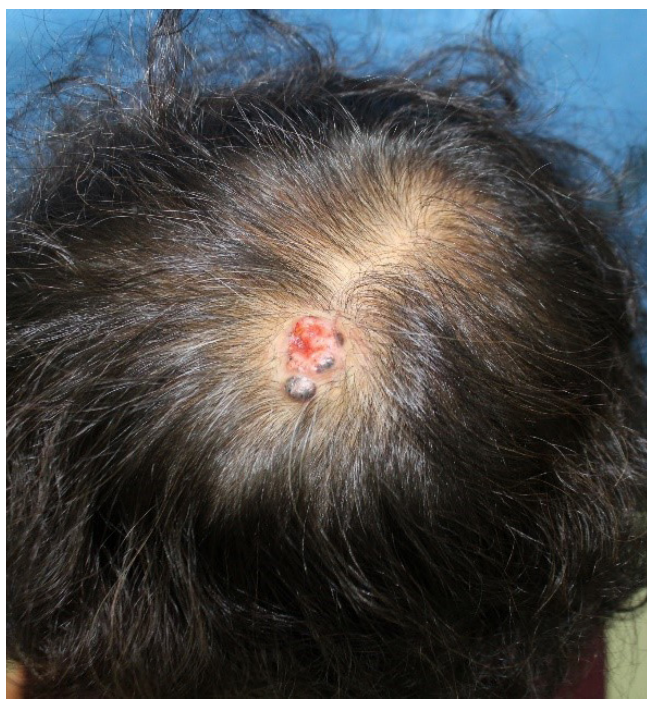

Fig. 1. Preoperative photograph showing a $2.0 \times 3.0 \times 0.5 \mathrm{~cm}$ round, protruding mass with ulceration and pigmented nodular lesions on the scalp.

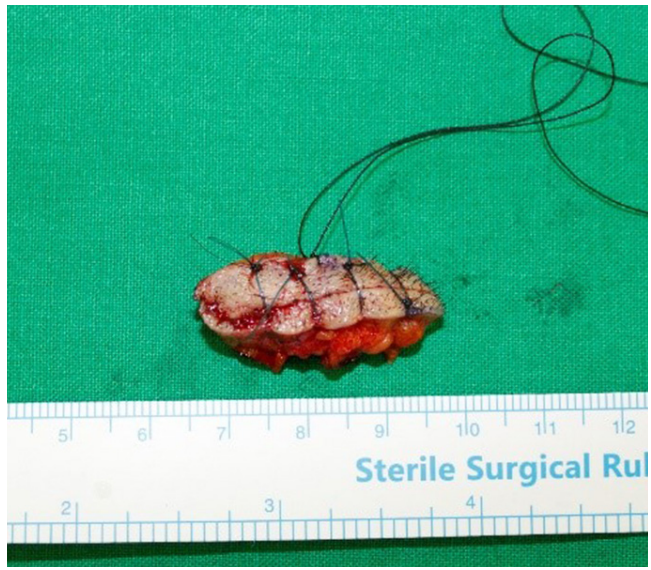

Fig. 2. Second excised specimen measuring about $3.0 \times 4.0 \times 1.0 \mathrm{~cm}$ in size.
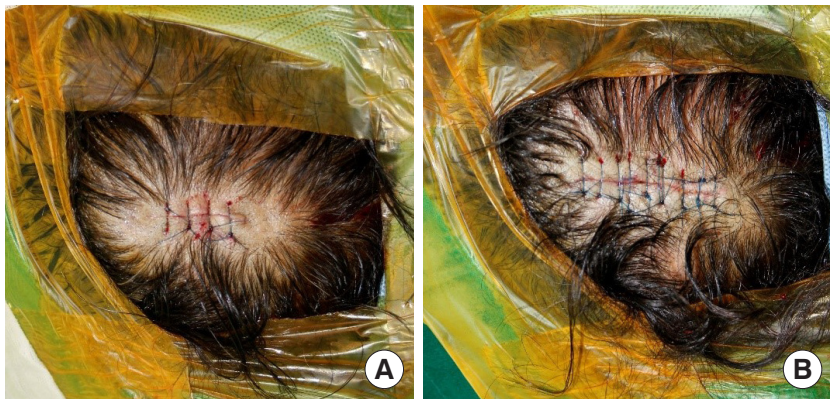

Fig. 3. Immediate postoperative photographs. (A) After the first excision. (B) After the second excision.

scribed neoplasm composed of small dark poroid cells and larger paler cuticular cells with clear cytoplasm and no connection to the overlying epidermis (Fig. 4).

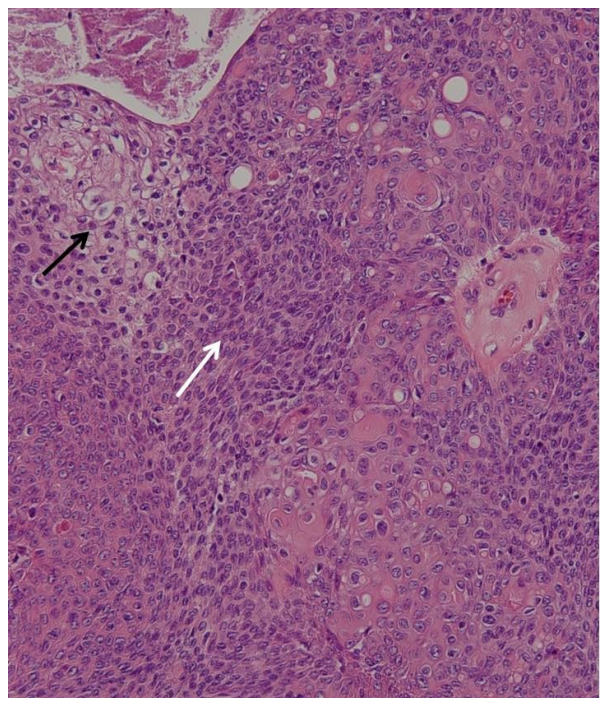

Fig. 4. Histologic examination. Solid tumor cell nests composed of small dark poroid cells (white arrow) and larger pale cuticular cells (black arrow) with clear cytoplasm $(\mathrm{H} \& \mathrm{E}, \times 100)$.

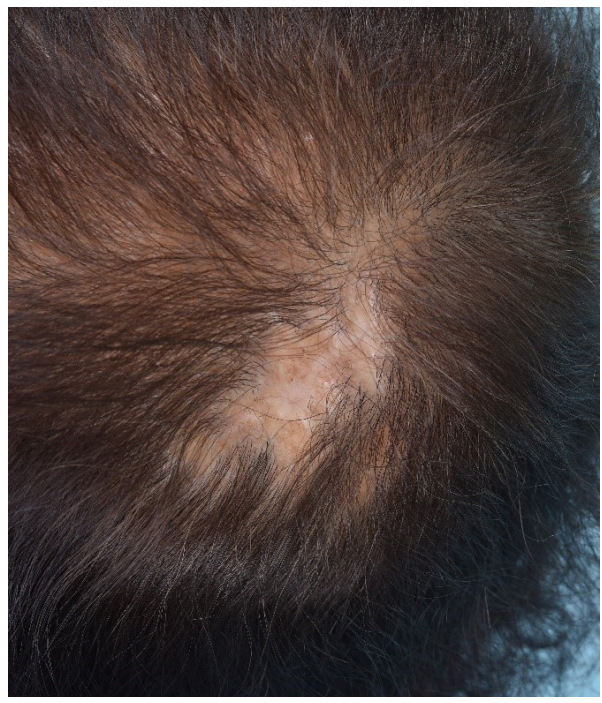

Fig. 5. Postoperative photograph. Surgical wound healed uneventfully leaving a scar and little alopecia as observed at the 1-year follow-up visit.

On immunohistochemistry, the tumor stained positive for high molecular weight cytokeratin and epithelial membrane antigen and negative for membrane-bound carcinoembryonic antigen, low molecular weight cytokeratin, CK20, and CD34.

At 1-year follow-up, she remains asymptomatic and without evidence of recurrence. The surgical wound healed uneventfully leaving only a scar and little alopecia (Fig. 5).

\section{DISCUSSION}

This case report of a poroid hidradenoma documents a rare 
finding in a medically complicated patient.

In 1990, Abenoza and Ackerman [1] described four poroid neoplasm variants according to neoplastic cell location: poroid hidradenoma, eccrine poroma, dermal duct tumor, and hidroacanthoma simplex. As these tumors derive from eccrine glands, variants are located entirely within the dermis [3]. Poroid hidradenoma is a tumor with solid and cystic components, where neoplastic poroid cells are all located within the dermis and without connection to the epidermis [4]. Eccrine poroma is a lesion with a clear margin between adjacent, normal epidermal keratinocytes and a population of smaller cuboidal cells, usually with darker nuclei protruding down into the underlying dermis [5]. The dermal duct tumor resembles to hidroacanthoma simplex but the tumor cells are located in the dermis. And hidroacanthoma simplex is characterized by nests of clearly discrete, small, rounded cells within the epidermis.

Despite the lack of reported malignancy with poroid hidradenoma, it can be developed to eccrine porocarcinoma as a primary lesion and be misdiagnosed with a malignant subcutaneous neoplasm and excised with a skin sparing procedure [6]. Further, it is not yet known whether additional safety margins should be used during excision.

We considered a high probability of pyogenic granuloma in the present patient because of the past history of caustic soda use and ulceration at the time of hospital visit.

Therefore, during surgery, frozen biopsies were performed to evaluate the possibility of skin cancer and once confirmed, a treatment plan to implement additional wide excision and evaluation was established.

As discussed above, the most common preoperative misdiagnoses for poroid hidradenoma are pyogenic granuloma or soft fibroma. There are difficult diagnoses correctly in clinical ground alone, because poroid hidradenoma originates from dermal tissue, therefore radical surgery is recommended.

As poroid hidradenoma is often a clinically misdiagnosed lesion, we recommend frozen biopsy and total mass excision, including the overlying skin and surrounding adipose tissue down to the superficial fascia, to prevent recurrence.

\section{NOTES}

\section{Conflict of interest}

No potential conflict of interest relevant to this article was reported.

\section{Ethical approval}

The study was approved by the Institutional Review Board of Bundang Jesaeng Hospital (IRB No. 2021-03-007) and performed in accordance with the principles of the Declaration of Helsinki. Written informed consent was waived.

\section{Patient consent}

The patient provided written informed consent for the publication and the use of her images.

\section{ORCID}

Byung Duk Min https://orcid.org/0000-0002-1498-6953

Chong Kun Lee https://orcid.org/0000-0002-6357-0444

Chang Eun Chung https://orcid.org/0000-0001-8706-1516

Dong Chul Kim https://orcid.org/0000-0003-3244-7918

So Ya Paik https://orcid.org/0000-0003-4707-574X

\section{Author contribution}

Conceptualization: BDM, CKL, CEC, DCK. Methodology: CEC, DCK, SYP. Project administration: CKL. Investigation: BDM, DCK, SYP. Resources: CKL, SYP. Supervision: CKL, CEC, DCK. Validation: CKL. Writing - original draft: BDM Writing - review \& editing: CKL.

\section{REFERENCES}

1. Abenoza P, Ackerman AB. Poromas. In: Abenoza P, Ackerman AB, editors. Neoplasms with eccrine differentiation. Philadelphia: Lea \& Febiger; 1990. p. 113.

2. Ito K, Ansai SI, Fukumoto T, Anan T, Kimura T. Clinicopathological analysis of 384 cases of poroid neoplasms including 98 cases of apocrine type cases. J Dermatol 2017;44:327-34.

3. Grant MT, Awad MM. Poroid hidradenoma of the scalp in an immunosuppressed patient. J Surg Case Rep 2019;2019:rjz146.

4. Requena L, Sanchez M. Poroid hidradenoma: a light microscopic and immunohistochemical study. Cutis 1992;50:43-6.

5. Penneys NS, Ackerman AB, Indgin SN, Mandy SH. Eccrine poroma: two unusual variants. Br J Dermatol 1970;82:613-5.

6. Seo BF, Choi HJ, Jung SN. Eccrine porocarcinoma on the cheek. Arch Craniofac Surg 2019;20:48-50. 